Article

\title{
Towards Stochasticity through Joint Invariant Functions of Two Isomorphic Lie Algebras of $S L(2 R)$ Type
}

\author{
Maricel Agop ${ }^{1,+}+\mathbb{D}$ and Mitică Craus ${ }^{2, *,+}$ (i) \\ 1 Department of Physics, Gheorghe Asachi Technical University, Iaşi 700050, Romania; m.agop@yahoo.com \\ 2 Department of Computers, Gheorghe Asachi Technical Univeristy, Iaşi 700050, Romania \\ * Correspondence: craus@tuiasi.ro \\ + These authors contributed equally to this work.
}

Received: 24 November 2019; Accepted: 15 January 2020; Published: 3 February 2020

check for updates

\begin{abstract}
In the motion fractal theory, the scale relativity dynamics of any complex system are described through various Schrödinger or hydrodynamic type fractal "regimes". In the one dimensional stationary case of Schrödinger type fractal "regimes", synchronizations of complex system entities implies a joint invariant function with the simultaneous action of two isomorphic groups of the $S L(2 R)$ type as solutions of Stoka type equations. Among these joint invariant functions, Gaussians become in the Jeans's sense, probability density (i.e., stochasticity) whenever the information on the complex system analyzed is fragmentary. In the two-dimensional case of hydrodynamic type fractal "regimes" at a non-differentiable scale, the soliton and soliton-kink of fractal type of the velocity field generate the minimal vortex of fractal type that becomes the source of all turbulences in the complex systems dynamics. Some correlations of our model to experimental data were also achieved.
\end{abstract}

Keywords: Lie groups; transformations; invariance; normalized Gaussian; stochasticity; uncertainty relation; fractal

\section{Introduction}

The theory of measurable Lie groups [1-3] is constituted as a fundamental procedure for integral geometry, necessary in generating the so-called geometric probabilities. The notion of geometric probability has a long history [4,5], except that recently [6-11] it has been explicitly realized that, by the theory of measurable groups, it could have applicability in physics or more generally, in fields involving statistical inference. The basis of this applicability lies in Jaynes' observation given in [12,13]. We will call it Jaynes' program for building geometric probabilities. According to this program, any circumstance left unspecified in a phenomenon has the concrete expression in the existence of an invariant group. Tats means that this circumstance is not left unspecified from subjective reasons, but because of the phenomenon under analysis, it is not felt at all. Obviously, statistical physics has never started from such premises in the construction of its concepts $[12,13]$. In such a context, the group theory [1-3] can be explained only through the idea that a continuous group is an invariant one for the results of some possible but non-actual constraints. We can justify this by examples that are detailed in this paper. As long as we limit ourselves to thermodynamic constraints, the quantity that we are interested in is the energy of the system in relation to which the distribution density typical of the system is an exponential function. When wanting a connection of the thermodynamics with the mechanics by which we study, for example, the harmonic oscillator (the entity of a complex system), the distribution density becomes, automatically, a Gaussian bivariate in impulse-coordinate [14]. 
The comparison of these distribution densities reveals that in the case of the exponential, the phase of the harmonic oscillator is lost. Therefore, the various elements of the thermodynamic system can be considered "replicas" of the same mechanical element. These "replicas" are obtained by measuring the coordinate and the impulse and they are characterized in the phase plane by different points grouped around the averages. Or, such points can be correlated between them by transformations of a measurable group $S L(2 R)$. The various achievements of this group will be considered in more detail in this paper, insofar as they can be related to statistical physics. In this conjecture, we will show that the density of such a distribution can be obtained as joint invariant function of two $S L(2 R)$ isomorphic Lie algebras, operating in a motion fractal theory $[10,11,15]$. Moreover, dynamics of complex systems at non-differentiable scale resolutions using various hydrodynamic type fractal "regimes" are analyzed.

\section{Short Reminder on the motion fractal theories in the Form of Scale Relativity}

The complex system is a set of entities (or structural units) that through their interactions, relationships, or dependencies form a unified whole. Let us give some examples: the polymer (or the co-polymer) is a complex system, its structural unit being the monomer; the laser ablation plasma also is a complex system, its structural units being the plasma particles; all biological structures are complex systems, their structural units being the cell, etc. [16-19].

The dynamics of complex systems can be described through various mathematical procedures (for details see $[17,18]$ ). If the complex system is assimilated to a fractal or multi-fractal (details on the importance of the fractal in nature, science, and engineering are given in [20-25]), a new mathematical procedure describing its dynamics is the scale relativity theory [15], an extension of this mathematical model being given in $[10,11]$.

Let us recall that the abovementioned mathematical models are included in motion fractal theories, the dynamics of these theories implying various mathematical procedures (for details see [23-27]). Moreover, the scale relativity theory in Nottale's sense [15] can be accepted as a particular case of the model presented in Appendix A for motion of complex system entities on Peano type curve (i.e., in fractal dimension $D_{F}=2$ ). For details, see also [10,11].

The fundamental assumption of the motion fractal theories in the form of scale relativity $[10,11,15]$, is the one that the dynamics of any complex system will be described by fractal motion curves. More details on motion fractal theories are given in the Appendix A.

In such a context, the dynamics of the complex system are given by means of scale covariance operator (scale covariant derivative) $\widehat{d} / d t$ (see the relation (A9) from the Appendix A and $[10,11]$ ):

$$
\frac{\widehat{d}}{d t}=\partial_{t}+\widehat{V}^{l} \partial_{l}+\frac{1}{4}(d t)^{\left(2 / D_{F}\right)-1} D^{l p} \partial_{l} \partial_{p}
$$

where

$$
\begin{aligned}
\widehat{V}^{l} & =V_{D}^{l}-V_{F}^{l} \\
D^{l p} & =d^{l p}-i \bar{d}^{l p} \\
d^{l p} & =\lambda_{+}^{l} \lambda_{+}^{p}-\lambda_{-}^{l} \lambda_{-}^{p} \\
\bar{d}^{l p} & =\lambda_{+}^{l} \lambda_{+}^{p}+\lambda_{-}^{l} \lambda_{-}^{p} \\
\partial_{t}=\frac{\partial}{\partial t^{\prime}}, \partial_{l}=\frac{\partial}{\partial X^{l}}, \partial_{l} \partial_{p} & =\frac{\partial}{\partial X^{l}} \frac{\partial}{\partial X^{p}}, i=\sqrt{-1}, l, p=1,2,3
\end{aligned}
$$

The meanings of the variables and parameters from relation (2) are given in Appendix A.

Under these conditions, applying the operator (1) to the complex velocity field from (2), the motion equations (i.e., the geodesics equation in a fractal space-see Appendix A) take the following form $[10,11]$ :

$$
\frac{\widehat{d} \widehat{V}^{i}}{d t}=\partial_{t} \widehat{V}^{i}+\widehat{V}^{l} \partial_{l} \widehat{V}^{i}+\frac{1}{4}(d t)^{\left(2 / D_{F}\right)-1} D^{l k} \partial_{l} \partial_{k} \widehat{V}^{i}=0
$$


For fractalization through Markov-type stochastic processes, the following relation results (Appendix A):

$$
\lambda_{+}^{i} \lambda_{+}^{l}=\lambda_{-}^{i} \lambda_{-}^{l}=2 \lambda \delta^{i l} .
$$

Under these conditions, the geodesics Equations (3) take the simple form

$$
\frac{\widehat{d} \widehat{V}^{i}}{d t}=\partial_{t} \widehat{V}^{i}+\widehat{V}^{l} \partial_{l} \widehat{V}^{i}-i \lambda(d t)^{\left(2 / D_{F}\right)-1} \partial^{l} \partial_{l} \widehat{V}^{i}=0 .
$$

For irrotational motions of the complex system entities, the complex velocity field $\widehat{V}^{i}$ takes the form:

$$
\widehat{V}^{i}=-2 i \lambda(d t)^{\left(2 / D_{F}\right)-1} \partial^{i} \ln \Psi,
$$

where $\Psi$ is the fractal state function.

Then, substituting (6) in (5) and using the method from [10,11], the geodesics Equation (5) becomes:

$$
\lambda^{2}(d t)^{\left(4 / D_{F}\right)-2} \partial^{l} \partial_{l} \Psi+i \lambda(d t)^{\left(2 / D_{F}\right)-1} \partial_{t} \Psi=0 .
$$

This is a Schrödinger equation of fractal type. Therefore, various dynamics of any complex system can be implemented as Schrödinger type fractal "regimes" (i.e., at various scale resolutions). For other correlations see $[28,29]$.

\section{Dynamics in a Complex System in the Form of Schrödinger Type Fractal "Regimes"}

In the one-dimensional stationary case, the Schrödinger equation of the fractal type takes the form (for details on the method, see [15]):

$$
\frac{d^{2} \Psi}{d x^{2}}+k_{0}^{2} \Psi=0
$$

with

$$
k_{0}^{2}=\frac{E}{2 m_{0} \lambda^{2}(d t)^{\left(4 / D_{F}\right)-2}} .
$$

In the above relations, $x$ is the fractal spatial coordinate, $E$ is the fractal energy of the entity, and $m_{0}$ is the rest mass of the entity.

In the most general case, $\Psi(x)$ is a complex function. Considering now that $\Psi(x)$ can be written in the form:

$$
\Psi(x)=X(x)+i Y(x),
$$

the Equation (8) becomes (in real variables):

$$
\begin{aligned}
& \frac{d^{2} X}{d x^{2}}+k_{0}^{2} X=0 \\
& \frac{d^{2} Y}{d x^{2}}+k_{0}^{2} Y=0 .
\end{aligned}
$$

Equations (11) and (12) are invariant to the group of transformations $S L(2 R)$ (for details see $[10,11])$.

$$
\begin{array}{r}
X^{\prime}=\alpha X+\beta Y \\
Y^{\prime}=\gamma X+\delta Y \\
\alpha \delta-\beta \gamma=1
\end{array}
$$

with $\alpha, \beta, \gamma$, and $\delta$ being real. 
The basis of the Lie algebra associated with the group (13) is given by the infinitesimal generators:

$$
X_{1}=Y \frac{\partial}{\partial X}, \quad X_{2}=\frac{1}{2}\left(X \frac{\partial}{\partial X}-Y \frac{\partial}{\partial Y}\right), \quad X_{3}=-X \frac{\partial}{\partial Y^{\prime}}
$$

and satisfies commutations relations

$$
\left[X_{1}, X_{2}\right]=X_{1}, \quad\left[X_{2}, X_{3}\right]=X_{3}, \quad\left[X_{3}, X_{1}\right]=-2 X_{2} .
$$

The most general solution of the Equation (11) (or (12)) is written in the form

$$
[X(x) \mid Y(x)]=z e^{i\left(k_{0} x+\theta\right)}+\bar{z} e^{-i\left(k_{0} x+\theta\right)},
$$

where $z$ is a complex amplitude, $\bar{z}$ is the complex conjugate of $z$, and $\theta$ is a specific phase. Thus, $z, \bar{z}$ and $\theta$ label each entity from an eventual complex system that has as a general characteristic the equation of state (11) (and (12)), and, consequently, the same $k_{0}$.

In such a conjecture, can one a priori establish a connection between the entities of the complex system? Since (16) has a "hidden" symmetry in the form of homographic group, we can answer this question affirmatively. Indeed, the ratio of two independent linear solutions of Equations (11) and (12), $\tau$, is a solution of Schwartz's differential equation [30]:

$$
\begin{array}{r}
\{\tau, x\}=\frac{d}{d x}\left(\frac{\ddot{\tau}}{\dot{\tau}}\right)-\frac{1}{2}\left(\frac{\ddot{\tau}}{\dot{\tau}}\right)^{2}=2 k_{0}^{2} \\
\dot{\tau}=\frac{d \tau}{d x}, \quad \ddot{\tau}=\frac{d^{2} \tau}{d x^{2}} .
\end{array}
$$

The left part of (17) is invariant with respect to the homographic transformation:

$$
\tau \leftrightarrow \tau^{\prime}=\frac{a_{1} \tau+b_{1}}{c_{1} \tau+d_{1}}
$$

with $a_{1}, b_{1}, c_{1}, d_{1}$ real parameters. The set of all transformations (18) corresponding to all possible values of these parameters is the group $S L(2 R)$.

Thus, all the entities of the complex system having the same $k_{0}$ are in biunivocal correspondence with the transformations of the group $S L(2 R)$. This allows the construction of a "personal" parameter $\tau$ for each entity of the complex system separately. Indeed, we choose as "guide" the general form of the solution of the Equation (17), which is written as

$$
\tau^{\prime}=u+v \tan \left(k_{0} x+\theta\right),
$$

where $u, v$ and $\theta$ are constants, and characterize an entity of the complex system.

By identifying the phase from (19) with one from (16), we can write the "personal" parameter of the entity as

$$
\tau^{\prime}=\frac{z+\bar{z} \tau}{1+\tau}, z=u+i v, \bar{z}=u-i v, \tau \equiv e^{2 i\left(k_{0} x+\theta\right)} .
$$

The fact that (20) is also a solution of the Equation (17) implies, by expliciting of (18), the group of transformations (for details see [9-11]):

$$
\begin{array}{r}
z^{\prime}=\frac{a_{1} z+b_{1}}{c_{1} z+d_{1}} \\
k^{\prime}=\frac{c_{1} \bar{z}+d_{1}}{c_{1} z+d_{1}} k .
\end{array}
$$


The infinitesimal generators of the group (21)

$$
B_{1}=\frac{\partial}{\partial z}+\frac{\partial}{\partial \bar{z}}, B_{2}=z \frac{\partial}{\partial z}+\bar{z} \frac{\partial}{\partial \bar{z}}, B_{3}=z^{2} \frac{\partial}{\partial z}+\bar{z}^{2} \frac{\partial}{\partial \bar{z}}+(z-\bar{z}) k \frac{\partial}{\partial k}
$$

with commutations relations

$$
\left[B_{1}, B_{2}\right]=B_{1},\left[B_{2}, B_{3}\right]=B_{3},\left[B_{3}, B_{1}\right]=-2 B_{2}
$$

reveals the same structure as the Lie algebra of the group $S L(2 R)$.

Thus, we can construct the joint invariant functions to the action of groups (13) and (21), as solutions of Stoka's equations [4-7]

$$
X_{i} Q+B_{i} Q=0, \quad i=1,2,3 .
$$

Explicitly, we will have

$$
\begin{array}{r}
Y \frac{\partial Q}{\partial X}+\frac{\partial Q}{\partial z}+\frac{\partial Q}{\partial \bar{z}}=0 \\
\frac{1}{2}\left(X \frac{\partial Q}{\partial X}-Y \frac{\partial Q}{\partial Y}\right)+z \frac{\partial Q}{\partial z}+\bar{z} \frac{\partial Q}{\partial \bar{z}}=0 \\
-X \frac{\partial Q}{\partial Y}+z^{2} \frac{\partial Q}{\partial z}+\bar{z}^{2} \frac{\partial Q}{\partial \bar{z}}+(z-\bar{z}) k \frac{\partial Q}{\partial k}=0
\end{array}
$$

The joint invariant functions have the form

$$
Q(\mu, v)=\text { const }
$$

with

$$
\mu=\frac{-i(z-\bar{z})}{(X-z Y)(X-\bar{z} Y)}, \quad v=k \frac{X-\bar{z} Y}{X-z Y}
$$

where $\mu$ is unimodular complex and $v$ is real.

A particular class of such joint invariant functions are the linear combinations of type:

$$
p \mu=m\left(v+\frac{1}{v}\right)+2 n
$$

with $m, n$ and $p$ real and arbitrary constants.

Taking into account (27), (28) becomes

$$
m k^{-1} L^{2}+2 n L \bar{L}+m k \bar{L}^{2}=p
$$

where

$$
L=\frac{X-\bar{z} Y}{[-i(z-\bar{z})]^{1 / 2}}
$$

under the condition

$$
-i(z-\bar{z})>0 .
$$

Relations (29) represent a family of conics from the plane $(X, Y)$. These are ellipses if

$$
m^{2}-n^{2}<0,
$$

a condition always satisfied if

$$
m=\sigma \sinh 2 r, \quad n=\sigma \cosh 2 r
$$

where $\sigma$ is a real constant. 
Thus, any joint invariant function is, here, a regular function of the algebraic expression (29).

Therefore, the group (21) works as a group of "synchronous" entities among the various entities of the complex system. In this process, the amplitudes and phases of each of them obviously participate, in the sense that they are also connected.

In what follows, let us notice that the structure of the group (21) is given by (23) so that the only non-zero structure constants should be:

$$
C_{12}^{1}=C_{23}^{2}=-1, C_{31}^{2}=-2
$$

Then, the invariant quadratic form is given by the "quadratic" tensor of the group (21)

$$
C_{\alpha \beta}=C_{\alpha \nu}^{\mu} C_{\beta \mu}^{v}
$$

where the summation over the repeated indices is understood.

Using (34) and (35), the tensor

$$
C_{\alpha \beta}=\left(\begin{array}{ccc}
0 & 0 & -4 \\
0 & 2 & 0 \\
-4 & 0 & 0
\end{array}\right),
$$

meaning that the invariant metric of the group (21) has the form

$$
\frac{d s^{2}}{f}=\Omega_{0}^{2}-4 \Omega_{1} \Omega_{2}
$$

with $f$ an arbitrary constant factor and

$$
\Omega_{0}=-i\left(\frac{d k}{k}-\frac{d z+d \bar{z}}{z-\bar{z}}\right), \quad \Omega_{1}=\frac{d z}{(z-\bar{z}) k}, \quad \Omega_{2}=-\frac{k d \bar{z}}{(z-\bar{z})},
$$

three differential 1-forms, absolutely invariant through the group (21) (for details see [9-11]).

In these onditions the metric (37) becomes:

$$
\frac{d s^{2}}{f}=-\left(\frac{d k}{k}-\frac{d z+d \bar{z}}{z-\bar{z}}\right)^{2}+4 \frac{d z d \bar{z}}{(z-\bar{z})^{2}}
$$

A particular case is the one induced by means of the parallel transport of direction in Levy-Civita sense [30]. Then, in the space of variables $(z, \bar{z}, k)$, the differential 1-form $\Omega_{0}$ is

$$
\Omega_{0}=0,
$$

while in the space of variables $(u, v, \theta)$ is

$$
d \theta=-\frac{d u}{v} .
$$

Since through (40) or (41) and (39) is/are reduced to the Lobacewski plan metric in Poincaré representation:

$$
\frac{d s^{2}}{f}=\frac{d z d \bar{z}}{(z-\bar{z})^{2}}=\frac{d u^{2}+d v^{2}}{v^{2}}
$$

$\theta$ from (40) will define the angle of the parallel transport of direction in Levy-Civita sense (for details see [8-11]). Once accepted functionality (40), the infinitezimal generators of the group (21) become:

$$
\overline{B_{1}}=\frac{\partial}{\partial z}+\frac{\partial}{\partial \bar{z}}, \quad \overline{B_{2}}=z \frac{\partial}{\partial z}+\bar{z} \frac{\partial}{\partial \bar{z}}, \quad \overline{B_{3}}=z^{2} \frac{\partial}{\partial z}+\bar{z}^{2} \frac{\partial}{\partial \bar{z}}
$$


and satisfy the commutation relations:

$$
\left[\bar{B}_{1}, \bar{B}_{2}\right]=\bar{B}_{1}, \quad\left[\bar{B}_{2}, \bar{B}_{3}\right]=\bar{B}_{3}, \quad\left[\bar{B}_{3}, \bar{B}_{1}\right]=-2 \bar{B}_{2} .
$$

Thus, we can construct now the joint invariant functions to the actions of group (13) and (21) with the restriction (40) (or (41)) as solutions of Stoka's equations [4-7]:

$$
X_{i} \bar{Q}+\bar{B}_{i} \bar{Q}=0, i=1,2,3 .
$$

Explicitly, we have

$$
\begin{array}{r}
Y \frac{\partial \bar{Q}}{\partial X}+\frac{\partial \bar{Q}}{\partial z}+\frac{\partial \bar{Q}}{\partial \bar{z}}=0 \\
\frac{1}{2}\left(X \frac{\partial \bar{Q}}{\partial X}-Y \frac{\partial \bar{Q}}{\partial Y}\right)+z \frac{\partial \bar{Q}}{\partial z}+\bar{z} \frac{\partial \bar{Q}}{\partial \bar{z}}=0 \\
-X \frac{\partial \bar{Q}}{\partial Y}+z^{2} \frac{\partial \bar{Q}}{\partial z}+\bar{z}^{2} \frac{\partial \bar{Q}}{\partial \bar{z}}=0 .
\end{array}
$$

The rank of this system is three, and that is only one independent integral. This is

$$
\frac{X^{2}-(z+\bar{z}) X Y+z \bar{z} Y^{2}}{-i(z-\bar{z})} .
$$

Thus, any joint invariant function is here a regular function of the algebraic expression (47).

Among them, the Gaussians

$$
\omega(X, Y \mid z)=\frac{1}{2 \pi} \exp \left[-\frac{1}{2} \frac{X^{2}-(z+\bar{z}) X Y+z \bar{z} Y^{2}}{i(z+\bar{z})}\right]
$$

can have completely special meanings.

Thus, according to Jaynes's program [12,13], we may think of a probability of synchronization for an entity-entity of a complex system, or a probability of synchronization for an entity-complex system. In any of the above situations, (48) will have stochastic significance.

According to our previous results, another important consequence, which can be experimental proven, is presented in the following.

Let us now consider another group of $S L(2 R)$ type given by means of infinitesimal generators.

$$
\bar{H}_{1}=\frac{\partial}{\partial h}+\frac{\partial}{\partial \bar{h}^{\prime}}, \bar{H}_{2}=h \frac{\partial}{\partial h}+\bar{h} \frac{\partial}{\partial \bar{h}^{\prime}}, \bar{H}_{3}=h^{2} \frac{\partial}{\partial h}+\bar{h}^{2} \frac{\partial}{\partial \bar{h}^{\prime}}
$$

which satisfies the commutation relations

$$
\left[\bar{H}_{1}, \bar{H}_{2}\right]=\bar{H}_{1}, \quad\left[\bar{H}_{2}, \bar{H}_{3}\right]=\bar{H}_{3}, \quad\left[\bar{H}_{3}, \bar{H}_{1}\right]=-2 \bar{H}_{2} .
$$

Then, the Stoka system [4,5] for operators (43) and (49) takes the form

$$
\begin{array}{r}
\frac{\partial F}{\partial h}+\frac{\partial F}{\partial \bar{h}}+\frac{\partial F}{\partial z}+\frac{\partial F}{\partial \bar{z}}=0 \\
h \frac{\partial F}{\partial h}+\bar{h} \frac{\partial F}{\partial \bar{h}}+z \frac{\partial F}{\partial z}+\bar{z} \frac{\partial F}{\partial \bar{z}}=0 \\
h^{2} \frac{\partial F}{\partial h}+\bar{h}^{2} \frac{\partial F}{\partial \bar{h}}+z^{2} \frac{\partial F}{\partial z}+\bar{z}^{2} \frac{\partial F}{\partial \bar{z}}=0 .
\end{array}
$$


Let it be noted that this system has the rank three, so that only one independent integral exists. This is the cross ratio generated by means of the relation

$$
\frac{h-z}{h-\bar{z}}: \frac{\bar{h}-z}{\bar{h}-\bar{z}} \equiv \chi^{2}
$$

In above relation, $\chi$ is real, and we took the square in order to account for the fact that the cross ratio (52) is always positive.

Any joint invariant function, $F$, is a regular function of this ratio. In such a context, if $\chi \equiv \tanh \beta$, where $\beta$ is arbitrary, then $z$ is by (52) related to $h$ through the linear relation

$$
z=\bar{u}+\bar{v} h_{0}
$$

where

$$
\begin{array}{r}
h=\bar{u}+i \bar{v}, \quad i=\sqrt{-1} \\
h_{0}=-i \frac{\cosh \beta-e^{-i \alpha} \sinh \beta}{\cosh \beta+e^{-i \alpha} \sinh \beta} \\
\Delta \beta=0
\end{array}
$$

$\Delta$ is the Laplace operator and $\alpha$ is real.

The above result shows that, according to $[8,9,30]$, a synchronization through self-modulation in amplitudes between two entities of a complex system is established. Such experimental results are presented in [31-33]. All results previously presented show a new approach for describing dynamics of complex systems through the isomorphism of two $S L(2 R)$ type groups, one of the variables, and another of the initial conditions. The joint invariant functions imposed by means of the Stoka theorem allow not only "all possible dynamics" but also "all possible probability densities" associated to these dynamics. Thus, this way is different with respect to that given through fractional derivatives [26,27].

\section{Dynamic of Complex Systems in the Form of Hydrodynamic Type Fractal "Regimes"}

In (5), the separation of the complex system dynamics on scale resolutions implies both the law of conservation of the specific fractal momentum

$$
\frac{\widehat{d} V_{D}^{i}}{d t}=\partial_{t} V_{D}^{i}+V_{D}^{l} \partial_{l} V_{D}^{i}-\left[V_{F}^{l}+\lambda(d t)^{\left[2 / D_{F}\right]-1} \partial_{l}\right] \partial^{l} V_{F}^{i}=0,
$$

at differentiable scale resolutions, and the conservation laws of the specific momentum

$$
\frac{\widehat{d} V_{F}^{i}}{d t}=\partial_{t} V_{F}^{i}+V_{D}^{l} \partial_{l} V_{F}^{i}+\left[V_{F}^{l}+\lambda(d t)^{\left[2 / D_{F}\right]-1} \partial_{l}\right] \partial^{l} V_{D}^{i}=0
$$

at non-differentiable scale resolutions. From (55), it results that at differentiable scale resolutions, a specific fractal force "operates":

$$
f_{F}^{i}=\left[V_{F}^{l}+\lambda(d t)^{\left[2 / D_{F}\right]-1} \partial_{l}\right] \partial^{l} V_{F}^{i}
$$

induced by the velocity fields at a non-differentiable scale.

In the static case $\left(V_{D}^{i}=0, \partial_{t} V_{F}^{i}=0\right.$-for details see $\left.[15,25]\right)$, the explicit form of the velocity field can be shown through the functionality of "evolution" equations (hydrodynamic equation of fractal type):

$$
f_{F}^{i}=\left[V_{F}^{l}+\lambda(d t)^{\left[2 / D_{F}\right]-1} \partial_{l}\right] \partial^{l} V_{F}^{i}=0
$$




$$
\partial_{l} V_{F}^{l}=0 .
$$

The first of these equations corresponds to the canceling of specific fractal force. The second equation describe the incompressibility of the fractal fluid (see Appendix A).

It is difficult to obtain an analytical solution for Equations (58) and (59). However, in the case of a plane symmetry (in $\mathrm{x}, \mathrm{y}$ coordinates), we can obtain a such solution. For this purpose, let us consider the equation system (58), (59) in the form:

$$
\begin{gathered}
u \partial_{x} u+v \partial_{y} u=v \partial_{y y}^{2} u \\
\partial_{x} u+\partial_{y} v=0
\end{gathered}
$$

where we substituted

$$
V_{F x}=u(x, y), \quad V_{F y}=v(x, y), \quad v=\lambda(d t)^{\left[2 / D_{F}\right]-1} .
$$

We will use the similarities method [34] to solve the equations system (60), (61) with the conditions

$$
\lim _{y \rightarrow 0} v(x, y)=0 \quad \lim _{y \rightarrow 0} \frac{\partial u}{\partial y}=0, \quad \lim _{y \rightarrow \infty} u(x, y)=0
$$

and a constant flux moment per unit of depth

$$
q=\rho \int_{-\infty}^{+\infty} u^{2} d y=\text { const. }
$$

We obtain the field of velocities in the following form:

$$
\begin{gathered}
u=\frac{1.5\left(\frac{q}{6 \rho}\right)^{\frac{2}{3}}}{(v x)^{\frac{1}{3}}} \operatorname{sech}^{2}\left[\frac{0.5 y\left(\frac{q}{6 \rho}\right)^{\frac{1}{3}}}{(v x)^{\frac{2}{3}}}\right] \\
v=\frac{1.9\left(\frac{q}{6 \rho}\right)^{\frac{2}{3}}}{(v x)^{\frac{1}{3}}}\left\{\frac{y\left(\frac{q}{6 \rho}\right)^{\frac{1}{3}}}{(v x)^{\frac{2}{3}}} \operatorname{sech}^{2}\left[\frac{0.5 y\left(\frac{q}{6 \rho}\right)^{\frac{1}{3}}}{(v x)^{\frac{2}{3}}}\right]-\tanh \left[\frac{0.5 y\left(\frac{q}{6 \rho}\right)^{\frac{1}{3}}}{(v x)^{\frac{2}{3}}}\right]\right\} .
\end{gathered}
$$

The above equations are simplified by means of both non-dimensional variables and non-dimensional parameters:

$$
\begin{gathered}
X=\frac{x}{x_{0}}, \quad Y=\frac{y}{y_{0}}, \quad U=\frac{u}{w_{0}}, \quad V=\frac{v}{w_{0}}, \\
\xi=\frac{v}{v_{0}}, \quad v_{0}=\frac{y_{0}^{\frac{3}{2}}}{x_{0}}\left(\frac{q}{6 \rho}\right)^{\frac{1}{2}}, \quad w_{0}=\frac{1}{\left(y_{0}\right)^{\frac{1}{2}}}\left(\frac{q}{6 \rho}\right)^{\frac{1}{2}},
\end{gathered}
$$

where $x_{0}, y_{0}, w_{0}$ and $v_{0}$ are lengths, velocity, and "fractality degree" specific to the complex system dynamics. The normalized velocity field is obtained:

$$
\begin{gathered}
U=\frac{1.5}{(\xi X)^{\frac{1}{3}}} \operatorname{sech}^{2}\left[\frac{0.5 Y}{(\xi X)^{\frac{2}{3}}}\right] \\
V=\frac{1.9}{(\xi X)^{\frac{1}{3}}}\left\{\frac{Y}{(\xi X)^{\frac{2}{3}}} \operatorname{sech}^{2}\left[\frac{0.5 Y}{(\xi X)^{\frac{2}{3}}}\right]-\tanh \left[\frac{0.5 Y}{(\xi X)^{\frac{2}{3}}}\right]\right\} .
\end{gathered}
$$


Any of the Equations (59), (60), (63), and (64) specify the nonlinearity of the velocity fields. So, a fractal soliton (i.e., a soliton dependent on scale resolution) is for the velocity field across the Ox axis. "Mixtures" of fractal soliton-fractal kink (i.e., a kink dependent on scale resolution), are for the velocity fields across the Oy axis. The fractality of the complex system is "explained" through its dependence from scale resolutions (Figures 1 and 2). Details on the soliton, kink, and other nonlinear solutions are given in [35].

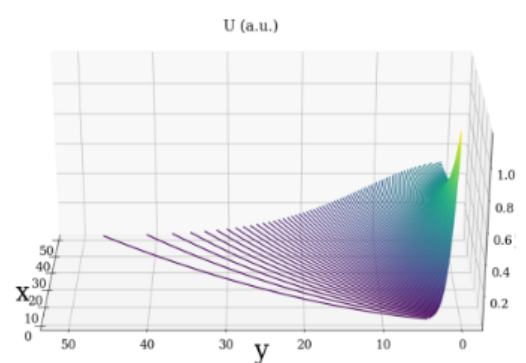

(a)

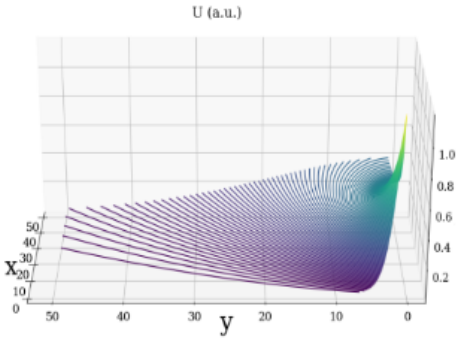

(b)

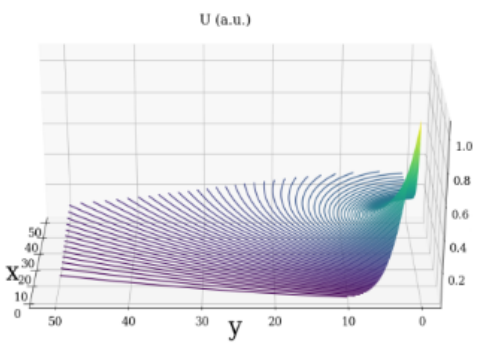

(c)

Figure 1. Normalized velocity field $U$ : (a) $\xi=0.4$, (b) $\xi=1$, (c) $\xi=2.5$.

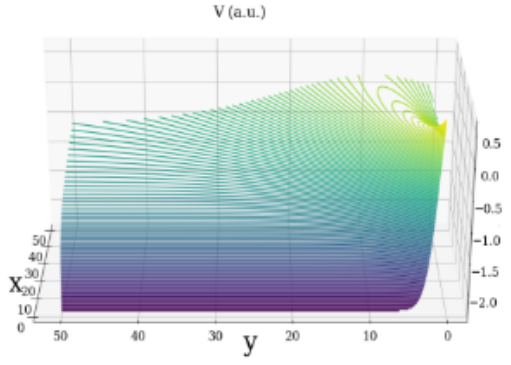

(a)

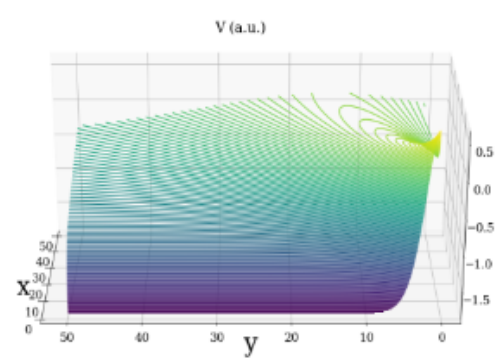

(b)

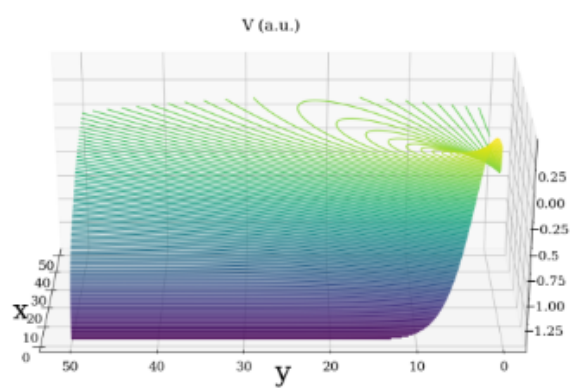

(c)

Figure 2. Normalized velocity field $V:$ (a) $\xi=0.4,(\mathbf{b}) \xi=1,(\mathbf{c}) \xi=2.5$. 
The velocity fields (69) and (70) induce the fractal minimal vortex (Figure 3).

$$
\begin{aligned}
\Omega= & \left(\frac{\partial U}{\partial Y}-\frac{\partial V}{\partial X}\right)=\frac{0.57 Y}{(\xi X)^{2}}+\frac{0.63 \xi}{(\xi X)^{\frac{4}{3}}} \tanh \left[\frac{0.5 Y}{(\xi X)^{\frac{2}{3}}}\right]+\frac{1.9 Y}{(\xi X)^{2}} \operatorname{sech}^{2}\left[\frac{0.5 Y}{(\xi X)^{\frac{2}{3}}}\right]- \\
& \frac{0.57 Y}{(\xi X)^{2}} \tanh ^{2}\left[\frac{0.5 Y}{(\xi X)^{\frac{2}{3}}}\right]-\left[\frac{1.5}{\xi X}+\frac{1.4 Y^{2}}{X(\xi X)^{\frac{5}{3}}}\right] \operatorname{sech}^{2}\left[\frac{0.5 Y}{(\xi X)^{\frac{2}{3}}}\right] \tanh \left[\frac{0.5 Y}{(\xi X)^{\frac{2}{3}}}\right]
\end{aligned}
$$

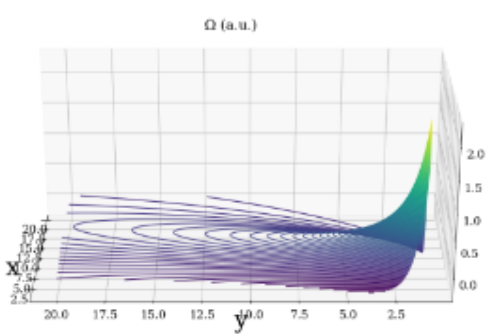

(a)

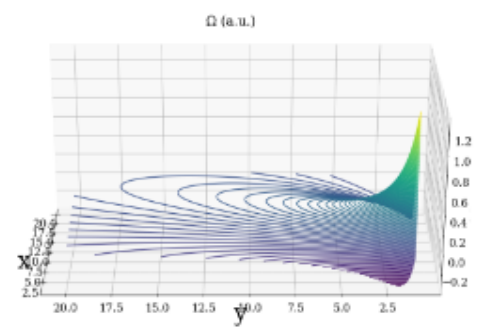

(b)

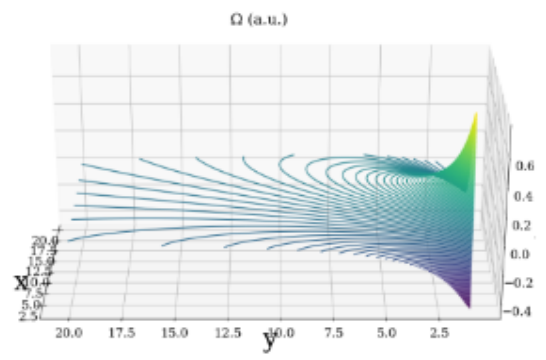

(c)

Figure 3. Fractal minimal vortex field $\Omega$ : (a) $\xi=0.4,(\mathbf{b}) \xi=1,(\mathbf{c}) \xi=2.5$.

The previous results were used to describe the dynamics of laser ablation plasma [31]. Moreover, the existence of the fractal minimal vortex (71) could explain the turbulent behaviors of the laser ablation plasma and of the atmosphere.

\section{Conclusions}

The main conclusions are the following:

(i) In the fractal theory of motion in the form of scale relativity, it is shown that in the case of the nonrotational movements of the entities of a complex system, its dynamics are associated with the geodesics on a fractal manifold in the form of Schrödinger equations at different scale resolutions. In such a context:

(i1) Explaining the dynamics of complex system entities in the form of one-dimensional stationary Schrödinger equations at various scale resolutions involves joint invariant functions at the action of two isomorphic groups of type $S L(2 R)$ (group of variables and parameters) as solutions of Stoka type equations. In such a context, special mechanisms for synchronizing the entities of a complex system become operable in the sense that not only the phases but the amplitudes of the complex system entities are homographically affected. 
(i2) The existence of a parallel transport of directions in the Levy-Civita sense, which specifies a certain amplitude-phase correlation for any entity of a complex system, explains a particular synchronization mechanism.

(i3) In any type of synchronization of the entities of a complex system, joint invariant functions of the Gaussian form become, according to Jeans's "program" [12,13], stochastic functions of probability density type (i.e., stochasticity), whenever information is fragmentary.

(i4) A special synchronization through self-modulation in amplitudes between two entities of the same type of a complex systems is established. Such results are proved in the dynamic of laser ablation plasma (the synchronization on the oscillation modes between the Coulomb structure and thermal structure).

(ii) In the fractal theory of motion in the form of scale relativity theory, it is shown that, in the general case, the dynamics in complex systems can be separated on the resolution scale (the differentiable and non-differentiable scales). Then, hydrodynamic type "regimes" to describe the dynamics become operational. In such a context:

(ii1) In the static case of motions at a non-differentiable scale resolution, the velocity fields are given by means of nonlinear solutions of fractal soliton type and fractal soliton kink-type.

(ii2) In the motion previous context, a fractal minimal vortex is generated. This case become the "source" of all turbulences in the dynamics of complex systems.

Author Contributions: All authors contributed equally to this work. They have read and agreed to the published version of the manuscript.

Funding: This research received no external funding

Conflicts of Interest: The authors declare no conflict of interest.

\section{Appendix A}

The fundamental hypothesis of the scale relativity theory $[10,11,15]$ is that the dynamics of the complex system are described through fractal curves. This leads to the following consequences [10,11,15]:

(i) Any fractal curve is explicitly scale resolution $\delta t$ dependent. More precisely, its length tends to infinity when $\delta t$ tends to zero (Lebesgue theorem [28]). Moreover, the space becomes a fractal in Mandelbrot sense [28].

(ii) The dynamics of complex systems structural units are described through fractal variables. Then, two derivatives of the variable field $Q(t, d t)$ can be defined:

$$
\begin{aligned}
& \frac{d_{+} Q(t, d t)}{d t}=\lim _{\Delta t \rightarrow 0_{+}} \frac{Q(t+\Delta t, \Delta t)-Q(t, \Delta t)}{\Delta t} \\
& \frac{d_{-} Q(t, d t)}{d t}=\lim _{\Delta t \rightarrow 0_{-}} \frac{Q(t, \Delta t)-Q(t-\Delta t, \Delta t)}{\Delta t}
\end{aligned} .
$$

The "+" sign corresponds to forward processes, while the "-" sign correspond to the backwards ones.

(iii) The differential of the spatial coordinate field $d_{ \pm} X^{i}(t, d t)$ is given by the expression:

$$
d_{ \pm} X^{i}(t, d t)=d_{ \pm} x^{i}(t)+d_{ \pm} \xi(t, d t) .
$$

The differential part $\left.d_{ \pm} x^{i}(t)\right)$ is scale resolution independent, while the fractal part $d_{ \pm} \xi(t, d t)$ is scale resolution dependent. 
(iv) The non-differentiable part of the spatial coordinate field satisfies the fractal equation $[10,11,15]$ :

$$
d_{ \pm} \xi^{i}(t, d t)=\lambda_{ \pm}^{i}(d t)^{1 / D_{F}}
$$

In (A3a) $\lambda_{ \pm}^{i}$ are constant coefficients associated to differentiable - non-differentiable transition and $D_{F}$ defines the fractal dimension of the non-differentiable curve.

We note that, in the general case, the non-differentiable part of the spatial coordinate satisfies the multifractal equation $[11,20-22,28]$ :

$$
d_{ \pm} \xi^{i}(t, d t)=\lambda_{ \pm}^{i}(d t)^{[2 / f(\alpha)]-1}
$$

where $\lambda_{ \pm}^{i}$ are constant coefficients associated to differentiable - non-differentiable transition, $f(\alpha)$ is the singularity spectrum of order $\alpha$, and $\alpha$ is the singularity index. There are many modes; hence, there is a varied selection of definitions of fractal dimensions: more precisely, the fractal dimension in the sense of Kolmogorov, the fractal dimension in the sense of Hausdorff-Besikovici, etc [28]. Selecting one of these definitions and operating in the complex system dynamics, the value of the fractal dimension must be constant and arbitrary for the entirety of the dynamical analysis. For example, we regularly find $D_{F}<2$ for correlative processes, $D_{F}>2$ for non-correlating processes, etc. In such a conjecture, through (A3b) we can identify not only the "areas" of the complex system dynamics that are characterized by a certain fractal dimension, but also the number of "areas" whose fractal dimensions are situated in an interval of values. More than that, through the singularity spectrum $f(\alpha)$ we can identify classes of universality in the complex system dynamic laws, even when regular or strange attractors have different aspect $[11,28]$.

(v) The differential time reflection invariance of any variable is recovered by means of the operator:

$$
\frac{\widehat{d}}{d t}=\frac{1}{2}\left(\frac{d_{+}+d_{-}}{d t}\right)-\frac{i}{2}\left(\frac{d_{+}-d_{-}}{d t}\right)
$$

This is a natural result of the Cresson's theorem [29]. Applying now the operator (A4) to X ${ }^{i}$, yields the complex velocity field:

$$
\widehat{V}^{i}=\frac{\widehat{d} X^{i}}{d t}=V_{D}^{i}-V_{F}^{i}
$$

with

$$
V_{D}^{i}=\frac{1}{2} \frac{d_{+} X^{i}+d_{-} X^{i}}{d t} \quad V_{F}^{i}=\frac{1}{2} \frac{d_{+} X^{i}-d_{-} X^{i}}{d t}, \quad i=1,2,3 .
$$

The real part $V_{D}^{i}$ is scale resolution independent, while the imaginary one $V_{F}^{i}$ is scale resolution dependent.

(vi) Since the fractalization implies stochasticization [28], the whole statistic arsenal in the form of averages covariances, etc., becomes operational. Thus, let us choose for the average of $d_{ \pm} X^{i}$ the following functionality:

$$
\left\langle d_{ \pm} X^{i}\right\rangle=d_{ \pm} x^{i}
$$

with

$$
\left\langle d_{ \pm} \xi^{i}\right\rangle=0
$$

The previous relation (A8) implies that the average of the non-differential part of the spatial coordinate field is null. 
(vii) The complex system dynamics can be described through the scale covariant derivative given by the operator:

$$
\frac{\widehat{d}}{d t}=\partial_{t}+\widehat{V}^{i} \partial_{i}+\frac{1}{4}(d t)^{\left(2 / D_{F}\right)-1} D^{l k} \partial_{l} \partial_{k}
$$

where

$$
\begin{array}{r}
D^{l k}=d^{l k}-i \bar{d}^{l k} \\
d^{l k}=\lambda_{+}^{l} \lambda_{+}^{k}-\lambda_{-}^{l} \lambda_{-}^{k}, \quad \bar{d}^{l k}=\lambda_{+}^{l} \lambda_{+}^{k}+\lambda_{-}^{l} \lambda_{-}^{k}
\end{array}
$$

For Markov-type stochastic processes [28]

$$
\lambda_{+}^{i} \lambda_{+}^{l}=\lambda_{-}^{i} \lambda_{-}^{l}=2 \lambda \delta^{i e},
$$

where $\lambda$ is a coefficient associated to the fractal-non-fractal transition, the scale covariant derivation (A9) becomes

$$
\frac{\widehat{d}}{d t}=\partial_{t}+\widehat{V}^{l} \partial_{l}-i \lambda(d t)^{\left(2 / D_{F}\right)-1} \partial^{l} \partial_{l}
$$

In the particular case of motions on Peano type curves, which implies $D_{F} \rightarrow 2$, the scale covariant derivative (A12) takes the standard form from the scale relativity theory [15]:

$$
\frac{\widehat{d}}{d t}=\partial_{t}+\widehat{V}^{l} \partial_{l}-i D \partial^{l} \partial_{l}
$$

where $\lambda \equiv D$ is the diffusion coefficient associated to fractal-nonfractal transition. Therefore, this model generalize all the results of Nottale's theory (i.e., Scale Relativity Theory [15]).

In such a context, when accepting the functionality of the scale covariance principle [15], i.e., applying the scale covariant derivatives in the form (A9), (A12), or (A13) to the complex velocity fields (A5), geodesics equations in a fractal space are obtained (for details see $[10,11,15]$ ).

\section{References}

1. Cartan, E. La Théorie de Groupes Finis et Continus et la Géométrie Différentiele Traiteés par la Méthode du Répere Mobile; Gauthier-Villars: Paris, France, 1951.

2. Postnikov, I. Groupes et Algebres de Lie; Édition Mir: Moscow, Russia, 1985.

3. Simon, B. Representations of Finite and Compact Groups; American Mathematical Society: Providence, RI, USA, 1996.

4. Stoka, M.I. Integral Geometry; Publishing House of the Academy: Bucharest, Romania, 1967.

5. Stoka, M.I. Géométrie Intégrale; Memorial des Sciences Mathématiques; Gauthier Villars: Paris, France, 1968.

6. Leuci, M.; Pastor A.M. Families of Curves Measurable with Respect to the Affine Modular Group. Suppl. Ai Rend. Del Circ. Mat. Di Palermo 1994, 35, 171-201.

7. Mazilu, N. The Stoka Theorem, a Side Story of Phisics in Gravitation Field. Suppl. Ai Rend. Del Circ. Mat. Di Palermo 2004, 77, 415-440.

8. Mazilu, N.; Agop, M. At the Crossroads of Theories. Between Newton and Einstein-The Barbilian Universe; Ars Longa Publishing House: Iasi, Romania, 2010. (In Romanian)

9. Mazilu, N.; Agop, M. Skyrmions: A Great Finishing Touch to Classical Newtonian Philosophy; World Philosophy Series; Nova: New York, NY, USA, 2012.

10. Mercheş, I.; Agop, M. Differentiability and Fractality in Dynamics of Physical Systems; World Scientific: Singapore, 2016.

11. Agop, M.; Merches, I. Operational Procedures Describing Physical Systems; CRC Press, Taylor \& Francis Group: Boca Raton, FL, USA, 2019.

12. Jaynes, E.T. Information Theory and Statistical Mechanics. Phys. Rev. 1957, 106, 620-630. [CrossRef]

13. Jaynes, E.T. The Well-Posed Problem. Found. Phys. 1973, 3, 477-493. [CrossRef]

14. Arnold, V.I. Mathematical Methods of Classical Mechanics; Springer: Berlin/Heidelberg, Germany, 1997. 
15. Notalle, L. Scale Relativity and Fractal Space-Time. A New Approach to Unifying Relativity and Quantum Mechanics; Imperial College Press: London, UK, 2011.

16. Bennett, C.H. How to Define Complexity in Physics and Why; In Complexity, Entropy, and the Physics of Information; Zurek, W.H. Eds.; Addison-Wesley Pub. Co.: Redwood City, CA, USA, 1990, pp. 137-148.

17. Bar-Yam, Y. Dynamic of Complex System. The Advanced Book Program; Addison-Wesley: Reading, MA, USA, 1997.

18. Badii, R.; Politi, A. Complexity: Hierarchical Structure and Scaling in Physics; Cambridge University Press: Cambridge, UK, 1997.

19. Mitchell, M. Complexity: A Guided Tour; Oxford University Press: Oxford, UK, 2009.

20. Wójcik, D.; Białynicki-Birula, I.; Zyczkowski, K. Time Evolution of Quantum Fractals; PRL85, 5022; The American Physical Society: College Park, MD, USA, 2000.

21. Aharony, A.; Feder, J. (Eds.) Fractals in Physics: Proceedings of the International Conference Honoring Benoit B. Mandelbrot on His 65th Birthday. Venice, France, Physica (Amsterdam) 38D, 1989; North-Holland: Amsterdam, The Netherlands, 1990.

22. Meakin, P. Fractals, Scaling and Growth Far from Equilibrium; Cambridge University Press: Cambridge, UK, 1998.

23. El-Nabulsi, R.A. Modifications at Large Distances from Fractional and Fractal Arguments. Fractals 2010, 18, 185-190. [CrossRef]

24. Parvate, A.; Gangal, A.D. Calculus on Fractal Subsets of Real LineI: Formulation. Fractals 2009, $17,53-81$. [CrossRef]

25. Amir-Azizi, S.; Hey, A.J.; Morris, T.R. Quantum Fractals. Compl. Syst. 1987, 1, 923-938.

26. Laskin, N. Fractional quantum mechanics and Lévy path integrals. Phys. Lett. A 2000, 268, $298-305$. [CrossRef]

27. Martins, J.; Ribeiro, H.V.; Evangelista, L.R.; da Silva, L.R.; Lenzi, E.K. Fractional Schrödinger equation with noninteger dimensions. Appl. Math. Comput. 2012, 219, 2313-2319. [CrossRef]

28. Mandelbrot, B. The Fractal Theory of Nature; Freeman: San Francisco, CA, USA, 1982.

29. Cresson, J.; Adda, F.B. Quantum Derivations and Schrödinger Equations. Chaos Solitons Fractals 2004, 19, 1323-1334.

30. Mihaileanu, N. Complements of Projective and Differential Analytical Geometry (in Romanian); Didactic and Pedagogical Publishing House: Bucharest, Romania, 1971.

31. Enescu, F.; Irimiciuc, S.A.; Cimpoesu, N.; Bedelean, H.; Bulai, G.; Gurlui, S.; Agop, M. Investigations of Laser Produced Plasmas Generated by Laser Ablation on Geomaterials. Experimental and Theoretical Aspects. Symmetry 2019, 11, 1391. [CrossRef]

32. Irimiciuc, S.A.; Bulai, G.; Gurlui, S.; Agop, M. On the separation of particle flow during pulse laser deposition of heterogeneous materials-A multi-fractal approach. Powder Technol. 2018, 339, 273-280. [CrossRef]

33. Irimiciuc, S.A.; Bulai, G.; Agop, M.; Gurlui, S. Influence of laser-produced plasma parameters on the deposition process: In situ space-and time-resolved optical emission spectroscopy and fractal modeling approach. Appl. Phys. A 2018, 124, 615. [CrossRef]

34. Schlichting, H. Boundary Layer Theory; McGraw-Hill: New York, NY, UISA, 1970.

35. Cristescu, C.C. Nonlinear Dynamics and Chaos: Theoretical Fundamentals and Applications; Romanian Academy Publishing House: Bucharest, Romania, 2008.

(C) 2020 by the authors. Licensee MDPI, Basel, Switzerland. This article is an open access article distributed under the terms and conditions of the Creative Commons Attribution (CC BY) license (http://creativecommons.org/licenses/by/4.0/). 